\title{
Influence of RNA Interference Targeting Rab5a on Proliferation and Invasion of Breast Cancer Cell Line MCF-7
}

\author{
Nier Cha ${ }^{1,2}$, Xinyue $\mathrm{Gu}^{1}$, Fengyun Yue ${ }^{5}$, Xiyao Wang ${ }^{3}$, Shusheng Jia ${ }^{1}$, Yuyan Ma ${ }^{4}$, Hongbin Wang ${ }^{1}$ \\ and Zhigao $\mathrm{Li}^{*}, 1$ \\ ${ }^{I}$ Department of Breast surgery, The Affiliated Third Hospital of Harbin Medical University, Harbin, Heilongjiang, \\ 150081, P.R. China \\ ${ }^{2}$ Department of Oncological Surgery, Inner Mongolia People's Hospital, Hohhot, Inner Mongolia, 010017, P.R. China \\ ${ }^{3}$ Department of surgery, The Hospital of Heilongjiang Women Prison, Harbin, Heilongjiang, 150081, P.R. China \\ ${ }^{4}$ Cancer Research Institute of Harbin Medical University, The Affiliated Tumor Hospital of Harbin Medical University, \\ Harbin, Heilongjiang, 150081, P.R. China \\ ${ }^{5}$ Faculty of Medicine, University of Toronto, Toronto, Ontario, Canada
}

\begin{abstract}
Objective: To explore the influence of RNA interference targeting Rab5a on proliferation and invasion of breast cancer cells, MCF-7, in vitro and provide a new direction for breast cancer gene therapy.

Method: Four miRNA interference vectors targeting Rab5a were designed, constructed, and transfected into breast cancer cell line MCF-7. RT-PCR and Western Blots were used to select the most efficient group of miRNA interference suppression. Next, the MTT and Transwell assays were applied to analyze cell proliferation inhibition rate and cell invasiveness.

Results: RT-PCR analysis showed that MCF-7 cells transfected with the Rab5a-miRNA-2 vector inhibited the Rab5a gene most strongly, with the inhibition rate being $(50.01 \pm 2.69) \%$. Western blot analysis also showed that cells transfected with Rab5a-miRNA-2 vector had the strongest inhibition of Rab5a protein, with the inhibition rate being $(55.58 \pm 3.16) \%$. Cells in this group were used Transwell assays. The Rab5a-miRNA-2 group was also used for MTT and Transwell assays. The MTT assay showed that the cell proliferation inhibitory rates were $(2.47 \pm 0.08) \%,(19.69 \pm 2.03) \%,(24.81 \pm 0.96) \%$, $(32.90 \pm 2.48) \%$ at $12 \mathrm{~h}, 36 \mathrm{~h}, 60 \mathrm{~h}, 84 \mathrm{~h}$ since Rab5a-miRNA-2 vector were transfected into MCF-7, respectively. There was a significant difference between the blank and negative controls $(\mathrm{P}<0.01)$. The Transwell assay showed that the number of cells passing through the membranes was $(67.8 \pm 12.03)$ at $48 \mathrm{~h}$ since transfection, with a significant difference between the negative controls and blank controls $(\mathrm{P}<0.01)$.
\end{abstract}

Conclusion: Rab5a can be silenced by miRNA expression vector thereby inhibiting the proliferation and invasiveness of MCF-7 breast cancer cells in vitro.

Keywords: RNAi, miRNA expression vector, Rab5a, breast cancer, cell proliferation, cell invasion.

Rab5a protein is a small GTP enzyme and a member of the Rab family, the largest branch of the Ras superfamily. As well, Rab5a is GTP switch located on the plasma membrane responsible for regulating the intracellular material transport, signal transduction, receptor down regulation, and phagocytosis of pathogens, among other functions. The Rab5a gene is located in 3p24.3 and its protein product is composed of 215 amino acid residues with a molecular weight of $23.658 \mathrm{kDa}$. A mutation in Rab5a leads to cell dysfunction, triggering diseases such as cancer. $\mathrm{Li} \mathrm{Yu}$ et al. found that Rab5a was highly expressed in human lung adenocarcinoma cell line Anip973 with high metastatic potential, and the opposite was shown in its low metastatic

*Address correspondence to this author at the Department of Breast Surgery, The Affiliated Tumor Hospital of Harbin Medical University, Harbin, Heilongjiang, 150081, P.R. China; Tel: 0086-13936458000;

E-mails: blake.e.z@gmail.com,drzhigaoli@126.com potential maternal AGZY83-a. The expression vector plasmid of Rab5a was transfected into AGZY83-a and cell morphology was seen to change to Anip973 by Stability screening [1], therefore suggesting an important role for the Rab5a gene during metastasis.

MicroRNAs (miRNAs), sized 19-25 nucleotides, are endogenous single-stranded RNA molecules existing widely in plants, animals, and viruses of eukaryotic cell biology. They are short RNAs and do not code for protein. The miRNA binds to target mRNA's 3'UTR bases thus inhibiting mRNA translation and producing post-transcriptional gene silencing for regulation of gene expression [2]. RNA interference (RNAi) is a gene silencing technology which is widely used in gene function studies. RNA interference technology applied to cancer gene therapy is an important field, as the expression of critical cancer genes related to tumor occurrence and malignant progression can be silenced. Studying the roles of the cancer genes to provide new 
biomarkers and molecular targets is important for gene therapy [3].

In this study we synthesized miRNA expression vectors of Rab5a to transfect breast cancer cell line MCF-7 in vitro. We investigated the change in cell biology behavior after lower Rab5a gene expression to explore the effect of the Rab5a gene on breast cancer cell growth, migration, and invasion, thereby providing a novel possibility for breast cancer gene therapy.

\section{MATERIALS AND METHODS}

\subsection{Building Rab5a miRNA Expression Vector}

The Rab5a gene sequence (Genbank: NM-004162) was used to design and synthesize four pairs of miRNA oligos and a pair of negative control oligos (RNAi design software, Invitrogen) (Table 1). Respectively, each pair of oligos were annealed into double-stranded miRNA and inserted into the pcDNA $^{\mathrm{TM}}$ 6.2-GW/EmGFPmiR expression vector (Invitrogen) to create recombinant clones (Vecmbintor construction kit, Invitrogen) and build four miRNA expression plasmids.

\subsection{Cell Culture and Transfection}

The human breast cancer cell line MCF-7 (Institute of Oncology, Harbin Medical University, Herbin, China) was cultured in medium supplemented with $10 \%$ fetal bovine serum. Exponential growth MCF-7 cells were seeded in 6well plates at a concentration of $4 \times 10^{5}$ cells per well until the degree of confluence was $80 \%-90 \%$ the following day. Four recombinant plasmids and a negative control plasmid were transfected into MCF-7 cells in 6-well plates according to the $\mathrm{FuGENE}^{\circledR}$ HD switch transfection reagent (Roche company) protocol. The concentration of plasmid DNA was $2.0 \mu \mathrm{g} /$ well prior to transfection, with $6.0 \mu \mathrm{l} /$ well of transfection reagent. FuGENE ${ }^{\circledR} \mathrm{HD}$ was added to cells in the final well as a blank control. $48 \mathrm{~h}$ after transfection the proportion of green fluorescent cells was observed using inverted fluorescence microscopy and transfection efficiency was determined. Finally, collected cells were trypsin-digested.

\subsection{Expression of Rab5a mRNA in MCF-7 Determined by RT-PCR}

Total RNA was extracted by the acid guanidium thiocyanate-phenol chloroform method with the total RNA extraction kit purchased from Waston (Shanghai). cDNA was prepared with reverse transcriptase (TaKaLa, Dalian) and 500ng of total RNA. The Rab5a primers (Upstream 5'CTTCAGAGGCAAGCAAGTCC-3', Downstream 5'-GTT GTGTGGGTTCGGTAAGG-3') gave a PCR product length of $268 \mathrm{bp}$. $\beta$-actin was used as a restricted reference material with primers: upstream 5'-CATCTCTTGCTCGAAGTCCA3', downstream 5'-ATCATGTTTGAG CCTTCAACA-3'. PCR products were separated by electrophoresis on a $2 \%$ agarose gel. Afterwards the gel image was captured using a UV-lamp camera and the expression levels of Rab5a mRNA were analyzed according to the absorbance of each band by LabWorks4.0 software.

\subsection{Rab5a Protein Expression in MCF-7 Measured by Western Blot}

Cells were collected from the six-well plates $48 \mathrm{hrs}$ after transfection and protein was extracted. Protein extract and buffer were mixed 4:1 after the protein concentration was measured. Twenty micrograms of protein was separated by sodium dodecyl sulfate-polyacrylamide gel electrophoresis and transferred to nitrocellulose membranes. The membranes were blocked with $3 \% \mathrm{BSA}$ for $1 \mathrm{~h}$ at room temperature followed by incubation with appropriate antibodies overnight at $4{ }^{\circ} \mathrm{C}$. The antibodies used were polyclonal rabbit antiRab5a antibody (1:1000, Santa Cruz Biotechnology) and polyclonal rabbit anti- $\beta$-actin antibody $(1: 3000$, Santa Cruz Biotechnology). Blots were probed with the primary antibodies, washed, and then incubated with horseradish peroxidase labeled secondary antibodies (1:10000, Beijign CoWin Biotech Co, Ltd). Binding was detected using enhanced chemiluminesence.

\subsection{Cell Proliferation Measured by the MTT Assay}

The Rab5a-miRNA interference vector and negative control plasmid from Group 2 were selected to transfect the MCF-7 cells according to method 1.2 and a group of cells with only transfection reagent added to form a blank control group. $5 \times 10^{3}$ cells were collected and resuspended in a $200 \mu \mathrm{l}$ volume in five wells of 96-well plates after $48 \mathrm{~h}$, followed by normal culturing in an incubator. After 12, 36, $60,84 \mathrm{~h}$ incubations, $20 \mathrm{ul}$ of $5 \mathrm{mg} / \mathrm{ml}$ solution of MTT was added to each well. The plates were then incubated for 4 hours at $37^{\circ} \mathrm{C}$. The precipitate was then solubilized in dimethylsulfoxide, 100ul/well, and shaken for 10 minutes. Absorbance (A) of each well was measured on an ELISA

Table 1. The Oligos of the miRNA and Negative Control

\begin{tabular}{|c|c|}
\hline Rab5a-miRNA-1-F & 5' TGCTGTTTATTTCCAGTATTTGGCCCGTTTTGGCCACTGACTGACGGGCCAAACTGGAAATAAA 3' \\
\hline Rab5a-miRNA-1-R & 5' CCTGTTTATTTCCAGTTTGGCCCGTCAGTCAGTGGCCAAAACGGGCCAAATACTGGAAATAAAC 3' \\
\hline Rab5a-miRNA-2-F & 5' TGCTGTTGATTTGCCAACAGCGGACTGTTTTGGCCACTGACTGACAGTCCGCTTGGCAAATCAA 3' \\
\hline Rab5a-miRNA-2-R & 5' CCTGTTGATTTGCCAAGCGGACTGTCAGTCAGTGGCCAAAACAGTCCGCTGTTGGCAAATCAAC 3' \\
\hline Rab5a-miRNA-3-F & 5' TGCTGAACTATGGCTGCTTGTGCTCCGTTTTGGCCACTGACTGACGGAGCACACAGCCATAGTT 3' \\
\hline Rab5a-miRNA-3-R & 5' CCTGAACTATGGCTGTGTGCTCCGTCAGTCAGTGGCCAAAACGGAGCACAAGCAGCCATAGTTC 3' \\
\hline Rab5a-miRNA-4-F & 5' TGCTGAACTATTGTCATCTGCATAGGGTTTTGGCCACTGACTGACCCTATGCATGACAATAGTT 3' \\
\hline Rab5a-miRNA-4-R & 5' CCTGAACTATTGTCATGCATAGGGTCAGTCAGTGGCCAAAACCCTATGCAGATGACAATAGTTC 3' \\
\hline Negative-F & 5' TGCTGAAATGTACTGCGCGTGGAGACGTTTTGGCCACTGACTGACGTCTCCACGCAGTACATTT 3' \\
\hline Negative-R & 5' CCTGAAATGTACTGCGTGGAGACGTCAGTCAGTGGCCAAAACGTCTCCACGCGCAGTACATTTC 3', \\
\hline
\end{tabular}



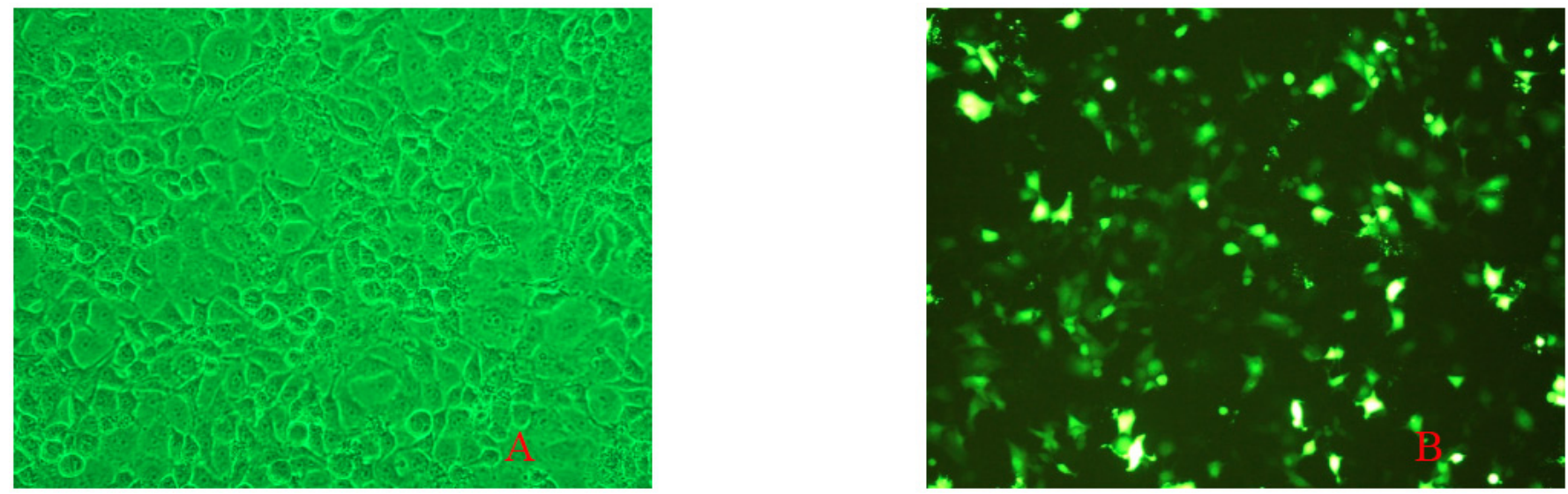

Fig. (1). Observation of transfection efficiency. Note: A: Optical microscope $(\times 100)$ B: Fluorescence microscope $(\times 100)$.

reader at a wavelength of $490 \mathrm{~nm}$, and a cell proliferation curve was drawn. The inhibitory rate of cell proliferation was calculated by the following formula: proliferation inhibition rate $(\%)=\left(1-\mathrm{A}_{\mathrm{RNAi}} / \mathrm{A}_{\text {Control group }}\right) \times 100 \%$.

\subsection{Cell Invasion Measured by the Transwell Assay}

In this study the membrane diameter was $6.5 \mathrm{~mm}$ with a pore size $8 \mu \mathrm{m}$. Transwell chambers (Corning Inc.) were placed in a 24-well plate and the surfaces were coated with $40 \mu 1$ Matrigel (1:8, BD Transduction). The bottom of the membrane was coated with $30 \mu \mathrm{l}$ fibronectin (Sigma Corporation) for $60 \mathrm{~min}$ at room temperature and left under UV lamp overnight. The Rab5a-miRNA interference vector and negative control plasmid of Group 2 were added to the MCF-7 cells according to method 1.2 and a group of cells had only transfection reagent added to act as a blank control group. After $48 \mathrm{~h}$, cells were collected and resuspended in RPMI 1640 without serum at a density $5 \times 10^{5} / \mathrm{ml}$ and then $200 \mu \mathrm{L}$ was transferred to the upper chamber. The lower chamber was filled with $600 \mu 110 \%$ fetal calf serum RPMI 1640 medium and each group was located three small chambers. After $24 \mathrm{~h}$ of incubation, the filter was gently removed from the chamber and the cells on the upper surface were removed by wiping with a cotton swab. Cells were washed with PBS twice, 95\% ethanol for 15 minutes, and stained with crystal violet. Cells were then counted in 5 randomly selected fields on a microscope $(\times 100)$ to calculate the average.

\subsection{Data Analysis}

All detection experiments were performed 3 times. All data are presented as means \pm SE. Comparisons were performed with ANOVA and LSD-T Test in SPSS.P $<0.05$ was considered statistically significant.

\section{RESULTS}

\section{1. miRNA Transfection Efficiency Calculated Using Fluorescence Microscopy}

Rab5a-miRNAs and negative control recombinant plasmids were transfected into human breast cancer cell line MCF-7. Successfully transfected cells showed green fluorescence expression under a fluorescence microscope after $48 \mathrm{~h}$. The number of green fluorescent cells was counted under a microscope and the plasmid transfection rate was found to be higher than $80 \%$ (Fig. 1).

\subsection{Inhibitory Effect of miRNA on mRNA Expression of Rab5a Gene}

RT-PCR results are shown in Fig. (2). $48 \mathrm{~h}$ after transfection the mRNA expressions of the Rab5a gene in the blank, negative control, and experimental groups are shown respectively in Table 2 . We found the inhibitory effects of Rab5a-miRNA-1, Rab5a-miRNA-2, and Rab5a-miRNA-4 were statistically significant $(\mathrm{P}<0.05)$. The strongest inhibitory effect was that of the Rab5a-miRNA-2 group, in which the inhibitory rate reaches $(50.01 \pm 2.69) \%(\mathrm{P}<0.001)$.

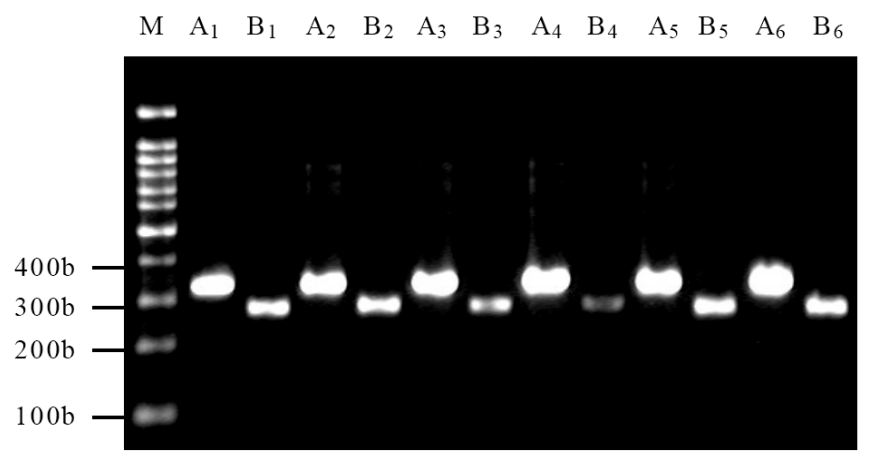

Fig. (2). The expression of Rab5a by RT-PCR. Note: M: marker A: $\beta$-actin B: Rab5a. 1: Blank control, 2: Negative control, 3: Rab5amiRNA-1, 4: Rab5a-miRNA-2, 5: Rab5a-miRNA-3, 6: Rab5amiRNA-4.

Table 2. mRNA Expression and Comparison of Rab5a in Cell Groups

\begin{tabular}{|c|c|c|}
\hline & mRNA Expression & P Value \\
\hline \hline Blank Control & $0.73 \pm 0.11$ & - \\
\hline Negative & $0.69 \pm 0.09$ & 0.480 \\
\hline Rab5a-miRNA-1 & $0.51 \pm 0.05$ & 0.003 \\
\hline Rab5a-miRNA-2 & $0.47 \pm 0.05$ & $<0.001$ \\
\hline Rab5a-miRNA-3 & $0.62 \pm 0.05$ & 0.083 \\
\hline Rab5a-miRNA-4 & $0.56 \pm 0.05$ & 0.012 \\
\hline
\end{tabular}




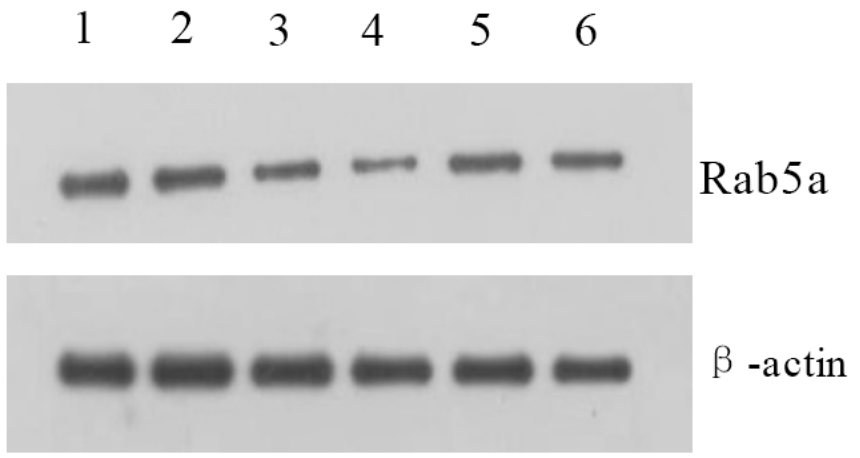

Fig. (3). Protein expression of Rab5a by Western Blot. Note: 1: Blank control, 2: Negative control, 3: Rab5a-miRNA-1, 4: Rab5amiRNA-2, 5: Rab5a-miRNA-3, 6: Rab5a-miRNA-4.

Table 3. Protein Expression and Comparison of Rab5a in Cell Groups

\begin{tabular}{|c|c|c|}
\hline & Protein Expression & P Value \\
\hline \hline Blank Control & $0.71 \pm 0.09$ & - \\
\hline Negative & $0.68 \pm 0.08$ & 0.607 \\
\hline Rab5a-miRNA-1 & $0.50 \pm 0.06$ & 0.007 \\
\hline Rab5a-miRNA-2 & $0.32 \pm 0.06$ & $<0.001$ \\
\hline Rab5a-miRNA-3 & $0.66 \pm 0.09$ & 0.446 \\
\hline Rab5a-miRNA-4 & $0.65 \pm 0.09$ & 0.393 \\
\hline
\end{tabular}

\subsection{Inhibitory Effect of miRNA on Rab5a Gene Protein Expression}

Western Blot results are shown in Fig. (3). 48h after transfection the protein expression of the Rab5a gene in the blank, negative control, and experimental groups are shown respectively in Table 3. We found the inhibitory effects of Rab5a-miRNA-1 and Rab5a-miRNA-2 were statistically significant $(\mathrm{P}<0.01)$. The effect of the Rab5a-miRNA-2 group was very significant $(\mathrm{P}<0.001)$, where the inhibitory percentage was $(55.58 \pm 3.16) \%$.

\subsection{Inhibitory Effect of Rab5a-miRNA-2 Recombinant Plasmid on Proliferation Activity of MCF-7}

Considering the above-mentioned RT-PCR and Western Blot experiments regarding Rab5a, the inhibitory effect exhibited by Rab5a-miRNA-2 was the strongest. Therefore,
Rab5a-miRNA-2 recombinant plasmid transfected MCF-7 cells were chosen to continue incubation for $12 \mathrm{~h}, 36 \mathrm{~h}, 60 \mathrm{~h}$, $84 \mathrm{~h}$. Using the MTT test, we found the difference between the negative control and the blank control groups was not statistically significant at each time point $(\mathrm{P}>0.05)$. However, the difference between the Rab5a-miRNA-2 group and the blank control group, as well as the difference between the Rab5a-miRNA-2 group and the negative control group were statistically significant $(\mathrm{P}<0.01)$ (Fig. 4). The inhibitory rate of proliferation activity due to Rab5amiRNA-2 interference of Rab5a was $(2.47 \pm 0.08) \%$, (19.69 $\pm 2.03) \%,(24.81 \pm 0.96) \%,(32.90 \pm 2.48) \%$ after $12 \mathrm{~h}, 36 \mathrm{~h}$, $60 \mathrm{~h}, 84 \mathrm{~h}$ incubation periods respectively.

\subsection{Inhibitory Effect of Rab5a-miRNA-2 Recombinant Plasmid on Invasive Ability of MCF-7}

$48 \mathrm{~h}$ after transfection the number of membranepenetrated cells in the negative control and blank control groups was $(148.2 \pm 9.12)$ and $(160.4 \pm 9.61)$ respectively, the difference between the two groups was not statistically significant. The number of membrane-penetrated cells in Rab5a-miRNA-2 group was $(67.8 \pm 12.03)$. The difference between the Rab5a-miRNA-2 and negative control groups was very significant. As well, the difference between the Rab5a-miRNA-2 group and the blank control group was also significant $(\mathrm{P}<0.01)$ (Fig. 5).

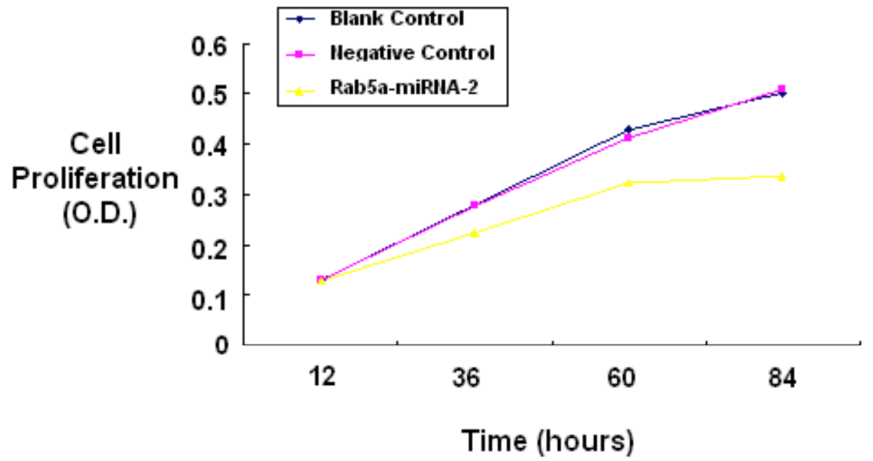

Fig. (4). MTT assay for proliferation of MCF-7 transfected by Rab5a-miRNA-2.

\section{DISCUSSION}

Rab5a protein is mainly present on the plasma membrane, clathrin-covered vesicles, and early endosomes. Not only is it related with cell material transport and protein sorting, but also with signal transduction, receptor downregulation, and phagocytosis of relevant pathogens. Studies
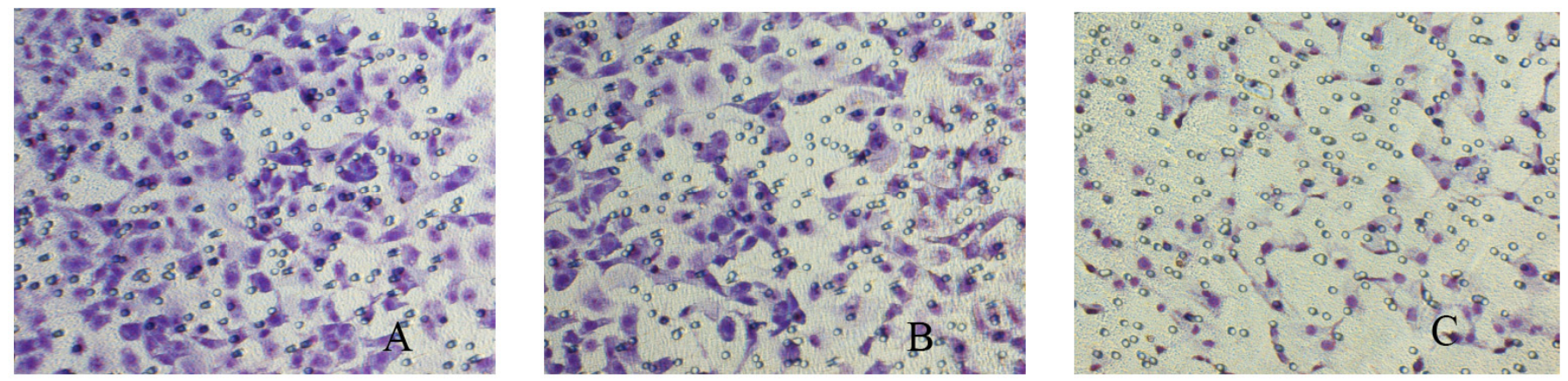

Fig. (5). Transwell assay for invasiveness of MCF-7 transfected by Rab5a-miRNA-2. Note: A: Blank control, B: Negative control, C: Rab5a-miRNA-2 $(\times 100)$. 
have shown that Rab5a protein mutations can lead to cell dysfunction and trigger immune deficiency, cancer, and other diseases. $\mathrm{Li} \mathrm{Y}$ et al. found that there was Rab5a overexpression in high metastatic potential human lung adenocarcinoma cell line Anip973 and low expression in its low metastatic potential cell lines AGZY83-a. Rab5a-ODN expression vectors were transformed into AGZY83-a cells and after screening cell morphology changed to Anip973 [1]. $\mathrm{Li} \mathrm{Z}$ et al. found that the Rab5a protein expression rate was $100 \%$ in colorectal cancer tissues with varying degrees of differentiation, its expression rate significantly increased with the degree of differentiation of colorectal cancer, and its expression rate increased with increased levels of colorectal cancer metastasis. Thus, it was suggested that Rab5a protein expression levels were closely related to invasion and metastasis of colorectal cancer [4].

RNA interference involves two types of small fragments of RNA, the siRNA and miRNA. The former is a long-chain dsRNA cut to $21 \sim 23$ nucleotides by the Dicer enzyme. siRNA, along with specific proteins and enzymes, form the RNA-induced silencing complex (RNA-induced silencing complex, RISC). The activated RISC is completely complemented with its homologous mRNA. The anti-sense siRNA-chain identified mRNA target fragment is cut by endonucleases, followed by degradation by exonuclease, and finally the expression of target genes are blocked [5]. As a class of non-coding small molecule RNA, miRNAs (microRNAs) have similar functions which regulate intracellular gene expression with siRNAs [6]. It is a long sequence of the original transcript (long primary transcript, pri-miRNA) and miRNA precursors (pre-miRNA) with a 70nt-generated stem-loop structure that is cut by Drosha in the nucleus, then digested to $21 \sim 22$ nucleotides of mature miRNA by the Dicer binding miRNP ribosome complexes to make miRNA-induced silencing complex (miRNAcontaining RNA-induced silencing complex, miRISC) [7]. Gene silencing of mature miRNA molecules is achieved by two ways. One way involves miRNA and the complementary target gene mRNA's 3'UTR blocking the translation of mRNA, but there is degradation because of incomplete pairing between mature miRNA and the target gene mRNA. The second way has similarity with the siRNA pathway, protein expression is blocked since target gene mRNA is degraded circularly by a positive feedback mechanism after miRNA complements completely with the homologous mRNA. Relative to siRNA, miRNA is more adept at gene silencing $[8,9]$.

In this study, we designed four pairs of miRNA gene precursor sequences for Rab5a, and cloned them into plasmid pcDNA ${ }^{\mathrm{TM}}$ 6.2-GW/EmGFPmiR to establish four RNA interference vectors which were transfected into human breast cancer cell line MCF-7. It confirmed that Rab5a was significantly inhibited by the interfering vector Rab5amiRNA-2 group in the levels of expression of gene and protein by RT-PCR and Western Blot, which was selected for follow-up experiments.

The occurrence, proliferation, invasion, and metastasis of tumor cells are extremely complex processes, and concern many factors including oncogenes, tumor suppressor genes, DNA damage repair genes, etc. We expected to determine the effect Rab5a on the breast cancer cells MCF-7 biological characteristics through the success of RNA interference suppression of Rab5a in gene expression and protein levels. We used the MTT assay to study correlation of Rab5a expression and proliferation of the breast cancer cells. The results show that the proliferation of MCF-7 in vitro was significantly lower than that of the negative control group when the Rab5a gene was suppressed by the interference vector of Rab5a-miRNA-2 group, and proliferation was reduced with time. We also used the Transwell assay to study the correlation of Rab5a expression and invasion of breast cancer cells. The results show that the number of trans-membrane cells was significantly reduced after the interfering vector of the Rab5a-miRNA-2 group was transfected into cells for $48 \mathrm{~h}$ compared to the negative and blank control groups, confirming that the invasion of MCF-7 breast cancer cell in vitro was significantly reduced with Rab5a inhibition.

Taken together these results suggest that Rab5a can provide a role for the proliferation and invasion of breast cancer cells. Inhibition by the miRNA expression vector on the expression of Rab5a could inhibit proliferation of breast cancer cell in vitro and weaken cell invasiveness. Therefore, these results indicate that Rab5a has implications in the treatment of breast cancer, however the molecular mechanism of Rab5a's effect on proliferation and invasion of breast cancer cells requires further investigation.

\section{REFERENCES}

[1] Li Y, Meng X, Feng H, Zhang G, Liu C, Li P. Over-expression of theRAB5 gene in human lung adenocarcinoma cells with high metastatic potential. Chin Med Sci J 1999; 14(2): 96-102.

[2] Wu L, Fan J, Belasco JG. MicroRNAs direct rapid deadenylation of mRNA. Proc Natl Acad Sci USA 2006; 103(11): 4034-9.

[3] Izquierdo M. Short interfering RNAs as a tool for cancer gene therapy. Cancer Gene Ther 2005; 12(3): 217-27.

[4] Zhi-Gao L, Xiao-Dong L, Xin-Shu D, Hong-Gang P, Jie D. Significance of RAB5A and CD44v9 expression in colorectal cancer tissues. World J Gastroenterol 2006; 14(9): 848-52.

[5] Mello CC, Conte D Jr. Revealing the world of RNA interference. Nature 2004; 431(7006): 338-42.

[6] Tang G. siRNA and miRNA: an insight into RISCs. Trends Biochem Sci 2005; 30(2): 106-14.

[7] Lee YS, Kim HK, Chung S, Kim KS, Dutta A. Depletion of human micro-RNA miR-125b reveals that it is critical for the proliferation of differentiated cells but not for the down-regulation of putative targets during differentiation. J Biol Chem 2005; 280(17): 16635-41.

[8] Nakahara K, Carthew RW. Expanding roles for miRNAs and siRNAs in cell regulation. Curr Opin Cell Biol 2004; 16(2): 127-33.

[9] Grimson A, Farh KK, Johnston WK, Garrett-Engele P, Lim LP, Bartel DP. MicroRNA targeting specificity in mammals: determinants beyond seed pairing. Mol Cell 2007; 27(1): 91-105. 